\title{
UAV LOW-COST SYSTEM FOR EVALUATING AND MONITORING THE GROWTH PARAMETERS OF CROPS
}

\author{
Maria Grazia D’Urso ${ }^{1, *}$, Andrea Rotondi², Mauro Gagliardini ${ }^{3}$ \\ ${ }^{1}$ DICeM, Department Civil and Mechanical Engineering, University of Cassino and Southern Lazio - mariagrazia.durso@unicas.it \\ ${ }^{2}$ PhD., DICeM - Via G. Di Biasio, 43 - 03043 Cassino (FR); +39 333 7676774; ing.rotondi@ yahoo.com \\ ${ }^{3}$ Fratelli Gagliardini, Operatori SAPR Rif. ENAC 5369, gagliardini.apr@gmail.com
}

Commission V, SS: Emerging Trends in Remote Sensing

key words: UAV, precision farming, vigour index , NIR, multi-spectral image, sensor, ortophoto

\begin{abstract}
New generation sensors (RGB, thermal, NIR and multispectral cameras) on unmanned aerial vehicles are continuously being developed for precision farming applications. In particular, this paper presents a study and an experimentation linked to the determination of viticulture growth indices by integrating the innovative techniques of digital photogrammetry from UAV platform, GPS satellite technique and traditional measures. Furthermore we demonstrate that the products from the processed raw-data represent good quality support to determine health state of the crop, and provide in addition a quantitative evaluation of the observed data. Through the use of such a technology it is possible to improve productivity between $15 \%$ and $17 \%$, reducing the market price of the product and the costs of control and management. In particular, during the monitoring campaign lasting three years from 2015 to 2017 , it was possible to observe that vine cultivation increased the growth index by about $12 \%$ following the corrections resulting from the critical interpretation of the results.
\end{abstract}

\section{INTRODUCTION}

The adoption of techniques and technologies for precision farming and, in particular, for precision viticulture, is currently an opportunity for botanists, producers and entrepreneurs in the industry to optimize the use of inputs in the production process by modulating agronomic interventions in a targeted manner while reducing environmental and health pressure.

In addition food safety and environmental conservation are two of the greatest challenges in the contemporary era. In relation to the global population growth forecast of around 9 billion by 2050 (Nikos and Bruinsma 2012), FAO estimates that increases of food and food products will rise by $60 \%$ if compared to the 2007 average. However, the globally cultivated area will grow to a negligible extent. In addition, factors such as technological evolution, socio-political changes, increased environmental degradation, water shortage, increased energy needs and the presence of new parasites and diseases are some current trends, different from the past, that affect agricultural production .

Increasing the size and structure of farms, automation strategies and climatic changes need to necessarily integrate technological innovation in a consolidated manner in the productive processes of modern agriculture. This is to address the challenges that will be faced in the coming decades, trying to make agriculture more productive and, at the same time, sustainable.

The need to improve productivity, competitiveness and environmental performance does not only concern the economy. With about 805 million people worldwide suffering from chronic malnutrition, most of which live in developing countries, Europe and the rest of the industrialized countries have the moral obligation to optimize agricultural production by boosting production in more sustainable way. Finally, the longterm challenges related to sustainable agriculture should be tackled by industrialized countries with a joint approach in order to support technological innovation, as well as a riskbased regulatory framework supported by scientific evidence,

\footnotetext{
${ }^{*}$ Corresponding author
}

continuity of basic and applied research and the development of skills in the agricultural field.

Recent investments and new funding priorities at Member State and EU level offer encouraging signals. The Horizon 2020 Framework Program is the largest EU research and innovation program, with around 80 billion euro available over the space of 7 years. New investments are being made in the Member States. For example, in the United Kingdom, the UK government is investing in a new Agri-Tech strategy aimed at making the UK the world's leading technology, innovation and sustainability of agriculture. Margins in the agricultural sector are reduced and additional costs threaten the survival of some small-scale activities. Finally, the long-term challenges of sustainable agriculture should be tackled with a joint approach by the Commission and the Member States to support technological innovation, a risk-based regulatory framework supported by scientific evidence, continuity of basic and applied research, and the development of skills in the agricultural field.

Within this framework UAV platforms equipped with low weight cameras and sensors offer flexible and low-cost tools for agricultural applications. Such systems, based on accurate measurements of the emitted and reflected energy, can monitor a wide range of crop parameters such as moisture content, surface temperature, photosynthesis activity and presence of weeds and parasites. This information is of growing importance to ensure the economic efficiency of agricultural production and harvest forecasts under a broader perspective of crop mitigation and adaptation to climate change and environmental sustainability.

\section{PRECISION AGRICULTURE}

The industrial agri-food system that has established itself over the last fifty years has led to indiscriminate exploitation, and irreversible deterioration, of natural resources faulty assumed to be unlimited and inexhaustible. The costs incurred in environmental and social terms due to intensive agriculture have been enormous, especially in terms of pollution, loss of biodiversity, soil fertility reduction, marginal land 
abandonment, thus creating obvious sustainability issues. Nowadays a possible response to the negative trend just described may be due to the development of technologies and the implementation of the so-called Precision Agriculture (PA), whose birth dates back to the nineties in the United States, where it continues to have the widest spread and technical and technological evolution. The term precision farming is associated with many others similar meanings, such as precision agriculture, site-specific management and prescription farming. At the same time, several and still evolving are the definitions of the term in the literature. Among the most common, we find the following expression: "That kind of agriculture that increases the number of decisions (correct), which are associated with net benefits, per unit area and per unit of time". The definition contains some fundamental aspects and, precisely, Precision Farming is defined as to: minimize costs; maximize productions; minimize environmental impacts (or optimize available environmental resources like water); use advanced technology to selectively intervene in crop and space. In North America, where farms compete aggressively both in the domestic and world markets, "Precision Farming" has already had a major development. Synthetically, we can summarize the technologies that are integrated as:

Remote sensing with UAV platform; GNSS Systems; Geographic Information Systems (GIS).

Currently, the application of this integrated methodology is particularly indicated in the wine-growing sector.

\subsection{Precision viticulture}

Precise farming applied to vineyards, Precision Viticulture (VP) involves the monitoring of physical, chemical and biological variations that provide the data needed to understand the real productivity of vineyards. The vast scientific research that is being done around the world aims to determine whether traditional sources of data acquisition (satellite and aerial photogrammetry) can support precision viticulture. Much of the research has focused attention on the ability to determine the general indexes obtained from the acquired images.

Cultivations and vegetation monitoring is highly dynamic, in particular that required by vineyard cultivation, which means that sensors on board of satellites in remote sensing are very limited due to the time needed to allow the next flight of the satellite on the same area. Moreover the vineyards, which are not a crop with a homogeneous distribution in the area, are difficult to observe by using the satellites, as their cameras cannot precisely distinguish the vegetation between the individual screws. This requires the use of photogrammetry from UAV platform using high resolution cameras.

Therefore, precision viticulture must necessarily be oriented to understanding the interactions between the vineyard and the environment in order to handle them to obtain quality grapes, which are the first requirement for excellent production. In viticulture, the management of agronomic practices such as fertilization, irrigation, pruning, processing, pesticide treatment requires not only a deep knowledge of plant physiology, but also of the territory and the environment.

This is particularly true in the light of the new environmental scenarios due to climate change that viticulture must cope with. The precision viticulture approach is therefore based on a continuous cyclical process that begins with observation of the vineyard performance and associated parameters, followed by the interpretation and evaluation of the collected data, until a targeted input management and / or selective grape harvest.

\subsection{Vigour indices}

In precision farming one often uses the spectral indices obtained as a simple algebraic combination of the measured spectral values at some specific wavelengths. They allow one either to synthesize the multispectral information in a single parameter related to the physiological and health status of the culture or to attenuate the disturbance of factors that similarly affect the different spectral bands (variations in lighting level, shading, effects due to the geometry of the vegetation, etc.). As the measurement techniques progressed, numerous spectral indices have been gradually introduced: among them the most used one is the Normalized Difference Vegetation Index (NDVI), defined by the ratio between the difference and the sum of reflectance in the near infrared and red:

$$
\mathrm{NDVI}=\left(\mathrm{NIR}_{780}-\mathrm{RED}_{680}\right) /\left(\mathrm{NIR}_{780}+\mathrm{RED}_{680}\right)
$$

This index is significantly related to the amount of plant biomass for unit surface (or Leaf Area Index, LAI), hence to the vigor of the crop. Its value varies from 0.1 to 0.3 , corresponding to a bare or little grassy soil, until reaching an asymptotic saturation value around $0.8-0.9$ for vegetation with $\mathrm{LAI}>5$. It is also possible to use other spectral indices, among which the one that seems to give the best benefits to identify particularly vigorous plants is the NDRE (Normalized Difference Red Edge - Normalized Difference in the Red band):

$$
\mathrm{NDRE}=\left(\mathrm{NIR}_{780}-\text { RedEdge }_{730}\right) /\left(\mathrm{NIR}_{780}+\text { RedEdge }_{730}\right)
$$

where the values 780 and $680 \mathrm{~nm}$ are indicative of bands centered on values of slightly different wavelenghts that allow one to compute the same NDVI index using several sensors. The NDRE index expresses the fact that the reflectance in the red band is, above all, influenced from the presence of chlorophyll and less biomass. The NDVI index has a linear response in the early stages of plant development, when the present biomass is limited but, viceversa, when the plant is in an advanced stage growth indicates that NDVI tends to be saturated by the high biomass content. On the contrary the NDRE index has a linear trend at all stages of growth. In particular the NDVI considerably changes between crops healthy and stressed at different stages of growth. However, as the development of the plant increases, the NDVI is no longer an effective indicator to distinguish the stressed and healthy plants because the relationship that binds the index to the vegetative vigour tends to flatten. The graph shows how NDRE is the best indicator to identify stronger and healthier plants than weaker ones, even in the most advanced stages of growth; this is because, even with high levels of vigour, one has a linear relationship between $\mathrm{x}$ and $\mathrm{y}$.

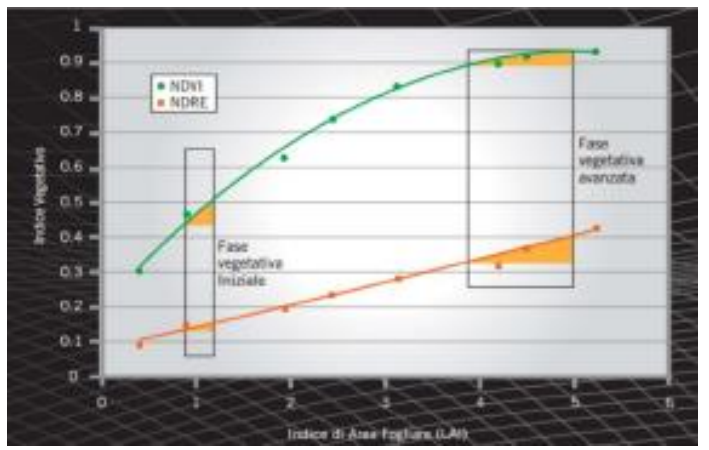




\section{Figure 1. Graph of the NDVI / NDRE strength indicators}

As it can be seen from the previous graph, the NDVI index tends to become saturated in the presence of very vigorous plants, viceversa the NDRE index always grows linearly even with high LAI values

\section{METHODS}

Since decades, the development and application of vegetation indices (VI) to monitor crop status has been in the focus of several research activities. Generally these VI are developed from multi-or hyper-spectral images of the field or remote sensing data.

In these last five years the image acquisition with UAVs has exponentially increased. If the multi and hyper-spectral sensors are still expensive and image capture and analysis require a high expertise (Aasen et alii 2015); it is nowadays possible to acquire geo-referenced RGB images with a very high spatial resolution by means of UAV platforms.

The RGB images from UAVs are very relevant for the research because this data source have many applications for precision farming. A well-established crop monitoring approach, which is also based on RGB images from UAV platforms, is the generation of multi-temporal Crop Surface Models (CSMs) for defining the plants height and the biomass estimations (Bending et alii, 2013). In this context tests and investigations were conducted by Bending et alii (2015) to analyze and evaluate the potential of RGB.

Bending et alii (2015) have also introduced a new, moderately performing, visible domain vegetation index, the RGBVI, which is applied in combination with the plant height from CSMs. Similar research are described by Bareth et alii (2015) and refer to the potential of the RGB vegetation indices. Both studies evaluated the RGBVI against the vegetation indices that were computed from hyper-spectral field spectrometer measurements.

Conversely the overall objective of this research is to further investigate the performance of the RGBVI from RGB images, NIR images, thermal and multi-spectral images captured with a low-cost UAV system. In this study the performance is not tested against field spectrometer measurements since this issue will be addressed in future research.

Therefore, four working packages are investigated below:

- Sensor 1: Carl Zeiss optics for RGB images

- Sensor 2: Canon S110 for NIR images

- Sensor 3: Flir thermal imaging

- Sensor 4: Tetracam ADC Micro for multi-spectral images. The ground-controlled UAV carrier or GCS mode, Gimbal Control Station, requires reliable communications from the aircraft to the control station and vice versa. In this experience a Skyrobotic ES - SRF SF6 has been used, with the following hardware configuration:

- 6 engines TIGER MOTOR MN 3508 380kV;

- 6 propellers TIGER MOTOR 14X4,8 CF PROPELLER;

- Autopilot: SR4000 MAIN rev 1.4 SKYROBOTIC;

- Powerboard: SR4000 rev 1.4 SKYROBOTIC; gimbal stabilized two axes - SIRALAB ROBOTICS; frame (frame + landing gear) SKYROBOTIC CARBON; SKYROBOTIC LiPo10900mA 22.2V batteries;

FTS (Flight Termination System) SKYROBOTIC with dedicated processor

The duration of each flight was 15 minutes on average, at an average speed of $2.5 \mathrm{~m} / \mathrm{sec}$ with each type of sensor. In order to verify the acquisitions, during the measurement campaign, a first download of country data and a quality check of the acquired images were made. This control became necessary because during the flight phase small wind bursts appeared. In particular, multispectral image captures were made at an altitude of about 52 meters, with a continuous acquisition rate of 1 second in order to ensure at least $60 \%$ overlap. The teflon tag image was acquired directly before the flight. The main purpose was to fix the coordinates of the GCPs (Ground Control Point) by means of white wooden panels of 50x50 cm in size and some of teflon tag taking into account all the problems arising from the atmospheric conditions and of the light conditions (such as the value of the incident angle of sunlight).

\section{SITE STUDY}

The vineyard object of study is located in the territory of the municipality of Anagni, in the province of Frosinone. The farm, whose coordinates are shown in the table, is located near the A1 motorway exit Anagni, about $45 \mathrm{~km}$ in the south of Rome.

\begin{tabular}{|l|ll|}
\hline WGS84 coordinates & \multicolumn{2}{|c|}{ '“ } \\
\hline Latitude & $41^{\circ} 43^{\prime}$ & 13.64 “ \\
Longitude & $13^{\circ} 06^{\prime}$ & 03.75 “ \\
\hline
\end{tabular}

Table 1. WGS84 Coordinates of the site

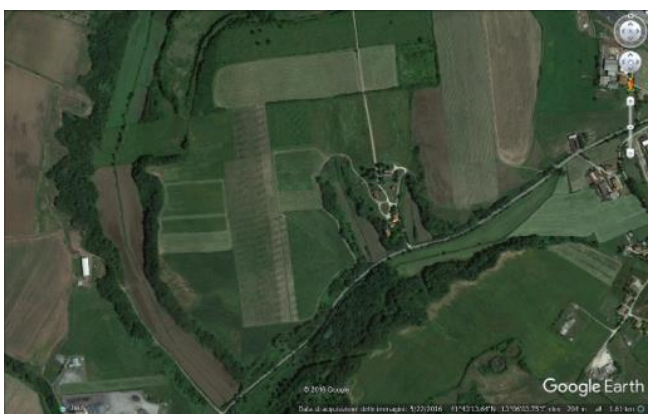

Figure 2. Monitoring area

The site is part of the geological structure of the Alban Hills.

This specific geographic position makes the chemical and physical composition of the soil to be volcanic (Vulcano Lazio, Latium Volcano), with the presence of red molten clay equal to almost $54 \%$ of the soil sample analyzed. The high amount of clay makes the soil poorly permeable with high water retention properties. From the chemical point of view, the soil contains potassium, calcium and minerals. The levels of nitrogen and phosphorus measured by property-commissioned analyzers are low. For what concerns the vineyards present in the farm, new cultivations were planted in 1962, following criteria that relate to ancient techniques. Weak or medium vigor rootstock have been implanted with high rigidity of the plant and with permanent filling of the interlayer to favor the development of the roots of vines at the expense of the grass roots. Such choices led to the limitation of the growing period of the shoots and the consequent elongation of the period of accumulation of sugars and noble compounds in berries. Among the vineyards, the predominant grape is the Cesane di Affile, a real viticultural and oenological jewel of this land as well as the native red grape native of Lazio. In addition, other red berries of international diffusion and traditional cultivation on our hills, such as Cabernet Sauvignon, Cabernet Franc, Petit Verdot, Merlot and Syrah are also grown. 


\section{MONITORING ACTIVITIES}

Cultivation growth index monitoring was performed through the elaboration of photogrammetric images from last generation drones equipped with photogrammetric sensors of the type: Thermal; RGB (visibile); NIR (Near Infrared); Multi-spectral. Thanks to the experience gained on the site under observation, acquisitions with such sensors were carried out through 6 flights in the months of June and September of the years 2015, 2016 and 2017.

The surface of the site, amounting to $150,000.00 \mathrm{~m}^{2}$ (15 ha), has made possible the use of UAVs with high flight range, in order to cover the entire plot of land.

\subsection{Monitoring activities in $\mathbf{2 0 1 5}$}

During the first year of tracking, several flights were carried out at various points using different photogrammetric cameras installed on board of a single Aibotix X6 aircraft. Once the system was calibrated, processing of images captured during 2 flights made in June and July 2015 with different cameras for RGB images and for NIR images, produced the following orthophotos (Fig.3).

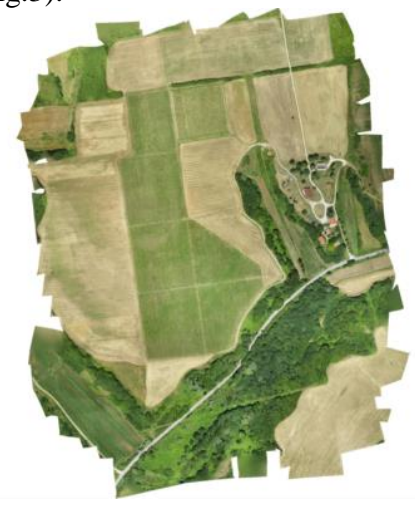

Figure 3. Orthophoto RGB June 2015

From the orthophotos generated in RGB mode it is possible to uniquely determine the extension of the vineyard and the adjacent crops. The vineyard appears to be of intense green color unlike the grain crops that have on a yellow tint (Fig.4).

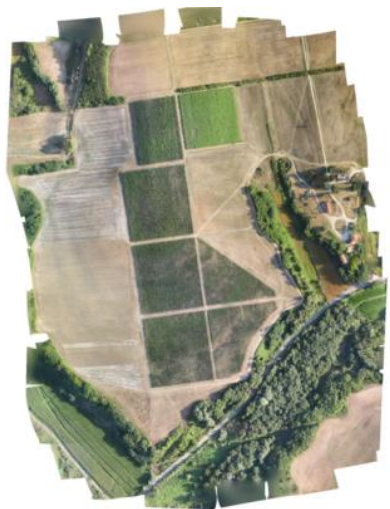

Figure 4. Ortophoto RGB

To determine the NDVI (Normalized Difference Vegetation Index) that can be calculated from the usable spectral information, further flights were performed with NIR image capture (Fig. 5) using a Canon PowerShot ELPH $110 \mathrm{Hs}$ camera with distance focal length of $4.3 \mathrm{~mm}$.

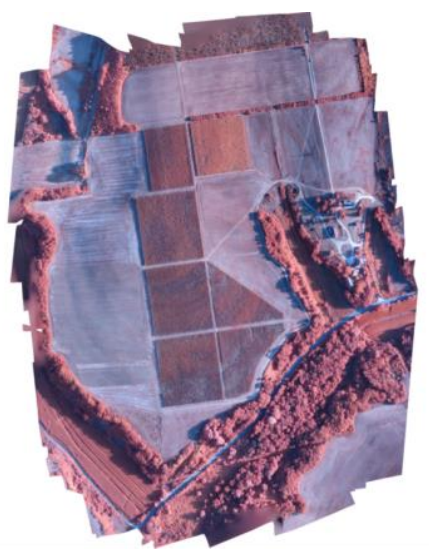

Figure 5. Ortophoto NIR

After having obtained the NDVI index maps for the months of June and July 2015, the average values of the NDVI index, identified by the pixels of the samples of the vineyards, have been those shown in the following table:

\begin{tabular}{|c|c|}
\hline June 2015 & July 2015 \\
\hline 0,541 & 0,637 \\
\hline
\end{tabular}

Table 2. NDVI indexes in 2015

\subsection{Monitoring activities in $\mathbf{2 0 1 6}$}

Based on the monitoring activities in 2015, the 2016 measurements campaign was carried out by using new sensors installed on the above mentioned UAV platform as well as an RGB camera and a NIR camera, including a thermal sensor and another multi-spectral sensor. In addition, as the new sensors were not compatible with the previously used drone, the UAV was changed to a Skyrobotic SF-6 esacotter.

The work platform has been implemented with ground-based remote control thus making possible to tune the flight plan via mobile Internet connection. Thanks to this type of remote control, the flight of the drone has been completely monitored on the ground: sending corrections of the flight and route parameters, the UAV aircraft has followed the overlay scheme shown in Figure 6.

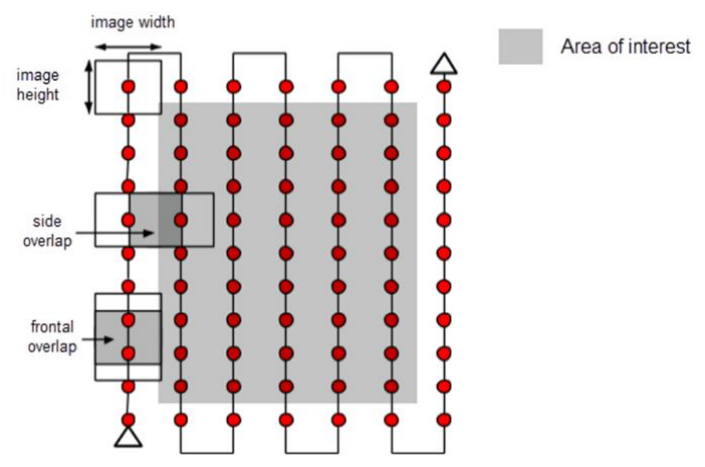

Figure 6. Overlapping scheme

The measurements were carried out on 27/07/2016 and 03/09/2016 from 12:00 to 13:00 in order to minimize the effect of shadows, so that the sun position is as vertical as possible. In both days atmospheric temperatures were greater than $30^{\circ} \mathrm{C}$, 
with peaks of $35^{\circ} \mathrm{C}$. Wind conditions were very favorable; only at the end of the last flight, when the UAV was installed on the multispectral sensor, the rise of a slight breeze forced the UAV driver to make small corrections of the flight plan.

Therefore, image acquisition has taken place, and Figure 7 shows an excerpt of the orthophoto RGB.

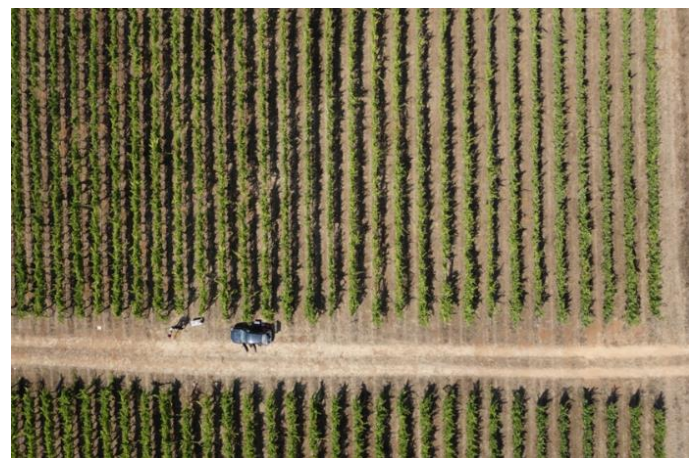

Figure 7. Partial RGB orthophoto

The image shown in Figure 7 was acquired in RGB mode at a flight height of 58.00 meters. The ground reprojection error was 1.72 pixels. The high degree of coverage and the image quality make it possible to clearly identify the targets that materialize the Ground Control Point (GCP) used as a topographic support network.

\subsubsection{Thermal sensor}

After flying in RGB mode, a first test was carried out, with a FLIR thermal sensor, in order to determine the temperature range of the vineyard. On the day of acquisition - 27/07/2016 the outdoor temperature was extremely high, close to $40^{\circ} \mathrm{C}$; hence some portions of the vineyard, especially those most exposed to sunlight, had an extremely high temperature gradient (Fig.8).

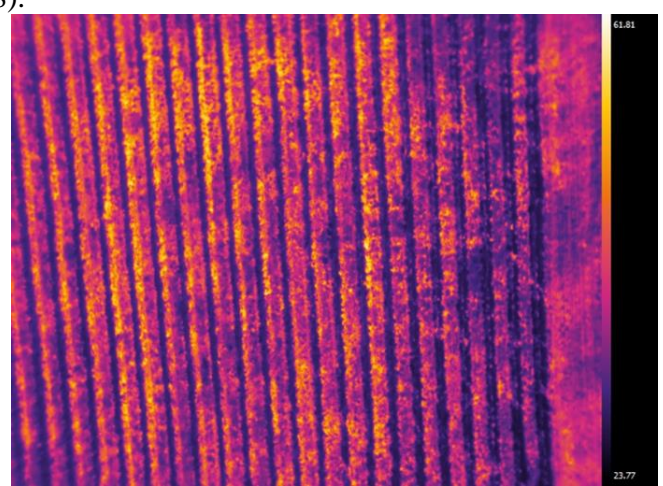

Figure 8. Acquisition by FLIR thermal camera

Some problems occurred in the elaboration of raw data. Particularly it has been impossible to elaborate a global orthophoto because the temperature gradient was variable between one photogram and another one so that the software couldn't compare pixels. Probably this fact has been due also to the strict proximity between the rows of the vineyard. Infact the same pixel presented two different colors in different photograms. This same problem occurred by analyzing the photograms in the grey scale, thus preventing to generate the global ortophoto. Hence, only the analysis of the single photogram has permitted to capture the zones characterized by a greater water critical issues, allowing for punctual interventions. Then, in addition to the locate the water stress, it has been also possible to locate the sandy-gravel zones where the ground is more drainage than the clay-silty zones where more waterproof layers are present. Infact in the zones of ground characterized by greater drainage capacity, the water evaporates rapidly and is wasted faster, emerging in the images as "warmer" than the ones where water evaporates and finds its way into the ground hardly (cold zone).

This very helpful information at the agronomic level has allowed the farmer to make timely irrigation in order to overcome the water shortage caused both by the geological characteristics and high temperature.

\subsubsection{Multi-spectral sensor}

In a further flight a TETRACAM ADC Micro multispectral sensor was used, with a particular mode of the acquisition of the spectrum bands, namely "rolling shutter" sensor, dealt in the paragraph 5.3

The image of Fig.9 shows a extract of the obtained multispectral ortho-images.

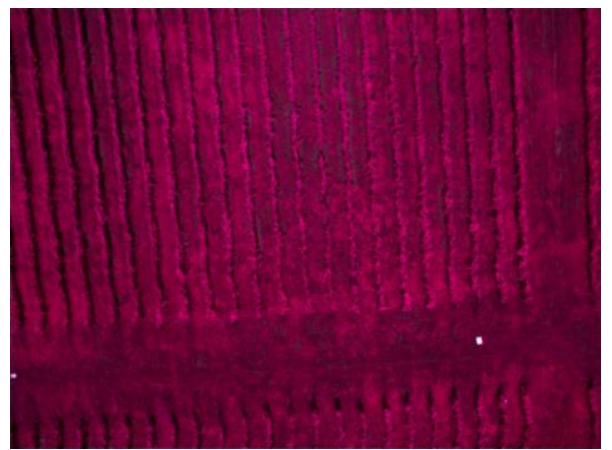

Figure 9. Multi-spectral orthophoto

Post-processing of the images allowed the NDVI index to be estimated for both measurement sessions in July and September.

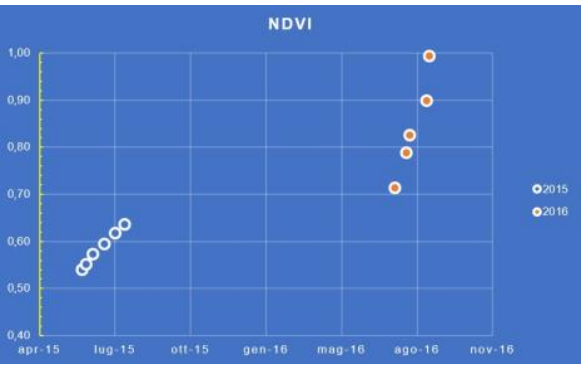

Figure 10. NDVI values 2015 - 2016

From a comparison of the obtained results it is possible to say that the NDVI value computed in July 2016 is about $12 \%$ greater than the one calculated in June 2015. This increase in the NDVI index is mainly due to the corrective inputs used by the farmer following the monitoring carried out after June 2015.

\subsection{Rolling shutter}

From the reading the camera's technical data sheet, it was found that the capture of images is carried out in the "Picture taking line" mode - the frame displays by line, and for the different color bands it was possible to infer the spectral response graph. 
The following figure, Fig. 11, which illustrates the spectral response graph for the several color bands, shows that the maximum responsiveness of the camera is in the green band and the red band, in the wavelengths contained in the spectrum of the visible.

Camera spectral response for red and green (blue is blocked for NIR Sensing):

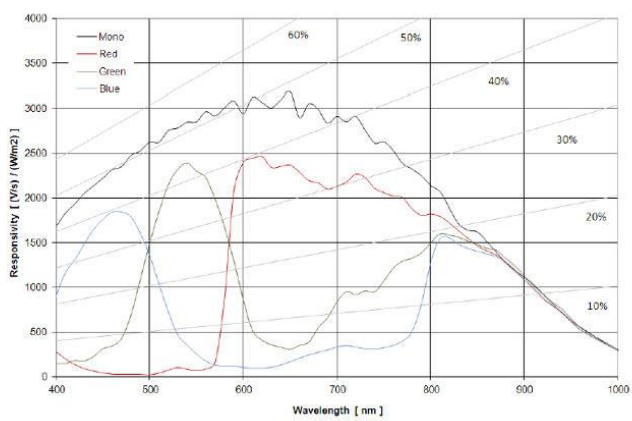

Figure 11. Spectral camera response for red and green

In few years the recent technological development has allowed one to obtain more advanced types of sensors equipped with an electronic mechanism known as "global shutter". For this kind of sensors all pixels contained in an image are captured at the same time. This means that the image will be "frozen", i.e. sharp without the blurred effect. The following chart shows a representative diagram relative to the different image capture mode with global shutter sensors and rolling shutter sensors.

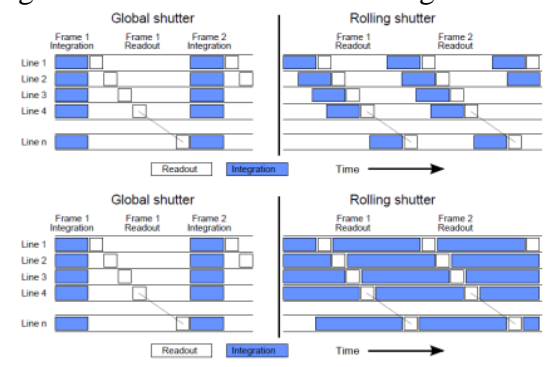

Figure 12. Different acquisition with global and rolling shutter

In the use of UAV platforms, the presence of a global shutter sensor has a great importance as the shutter is moving. It is easy to deduce the greater sharpness of the images, as well as the improved quality of the final processing.

The advantage of a "global shutter" room when it is used with the UAV platform is the ability to fly at an increasing speed without the risk of blurred and crisp images.

For example, in the Structure for Motion (SfM) field, cameras equipped with "global shutter" sensors, allows one to use only one image; vice-versa for cameras equipped with rolling shutter sensor multiple images are needed. This yields an increase in the computational burden due to a larger number of parameters to be estimated. However, the use of rolling shutter sensors is not precluded and one can continue to use them by performing a calibration process to make the "line delay" known.

The research activity that has been carried out allowed us to realize that using a rolling shutter sensor during field acquisition, with non-optimal climatic conditions, requires a speed flight lower with respect to the one typical of flights with a global shutter.

Although the flights with a rolling shutter sensor were carried out at a speed of about $3 \mathrm{~m} / \mathrm{s}$, we still faced the problem of the low sharpness since the images were acquired by lines.
In the future, it will be advisable to perform step-by-step tests to reduce UAV flight speed to minimize this effect on the photogram.

\subsection{Accuracy of the surveyed data}

To be able to estimate the accuracy of processed data detected through the use of the Agisoft Photoscan software a subdivision in sub - sectors, taken as sample areas, of the whole area occupied by rows of vineyards, has been considered The attention has been concentrated on the plots n. 3 and n. 4 represented in the Fig.13.

For each of them the precision data obtained have been deduced for the estimate of the NDVI index.

The values shown are representative of the individual sector.

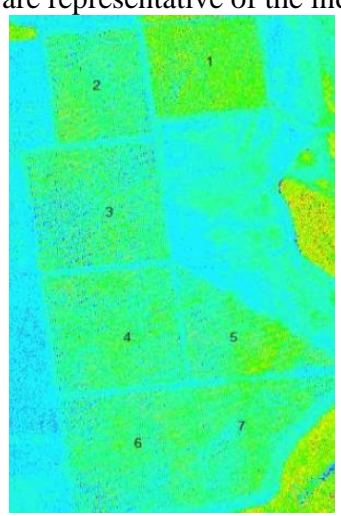

Figure 13. Study sectors

It's interesting to note that the values of standard deviation obtained on two different sectors are similar. This allows us to state that the acquisitions are accurate, and that the determined NDVI index values are representative of the health status of the vineyard.

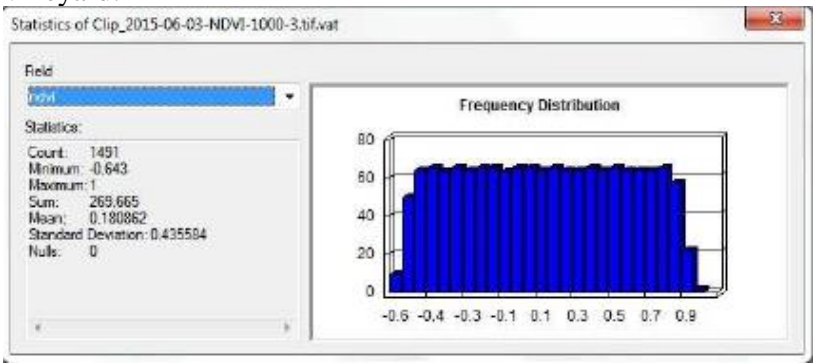

Figure 14. Sector 3 - Distribution frequency 03/06/2015

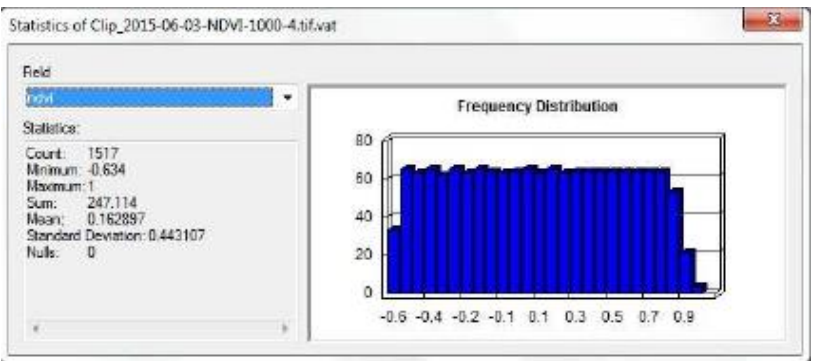

Figure 15. Sector 4 - Distribution frequency 03/06/2015 


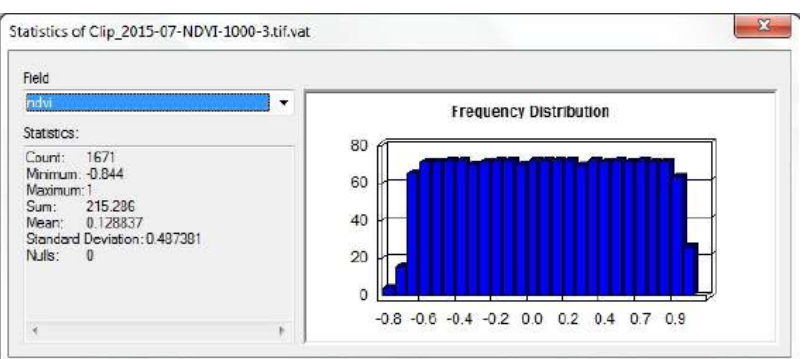

Figure 16. Sector 3 - Distribution frequency 10/07/2015

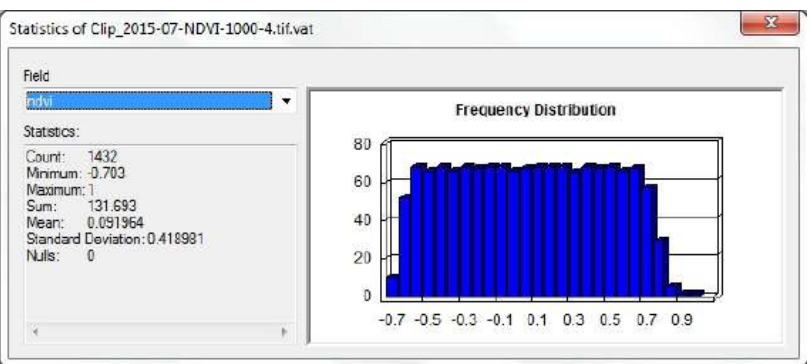

Figure 17. Sector 4 - Distribution frequency 10/07/2015

\subsection{Monitoring activities in 2017}

Monitoring activities carried out during the months July September 2017 enjoyed expertise and results of the monitoring activities over the years 2015 and 2016 years, with particular reference to overcoming the principal critical issue, affecting in particular the earlier measurement sessions, represented by the vibrations emitted from the vehicle using the multi-spectral camera. This inconvenience has been exceeded installing an optimized waster that can eliminate almost all emitted vibrations thus allowing us to achieve satisfactory results. The optimal set-up of the waster has been obtained by suitably modifying, during several tests, the stiffness of wasters installed on the inertial system of the vehicle. Several daily tests and experiences have been necessary to realize the Gimbal for the Micro Tetracam camera. In order to obtain very stable images a cardanic support with 2 axes, controlled by digital servo and automatically compensated by the giroscope which is linked to, regulates the horizontal and vertical tilt-shift of the support where the camera is installed.

In addition, the choice to decrease the navigation speed from $3 \mathrm{~m} / \mathrm{sec}$, used in the earlier monitoring activities, to the value 2.5 $\mathrm{m} / \mathrm{sec}$ has been very efficient in relation to the "rolling shutter". Infact, being the height of flight being equal to 70 meters, this has permitted Micro Tetracam to almost double the resolution, contrary to the ADC Tetracam snap with "global shutter" sensor. The flights dated 31/07/2017 and 02/09/2017, characterized by in stable environmental conditions, with temperature values of about $35^{\circ} \mathrm{C}$ degrees, in absence of wind and shadows, have permitted the acquisition of images using RGB and multi-spectral sensors. The ortophotos elaborated from multi-spectral images, reported in the sequel (Fig.18-21) for a sample area, highlight the good values of ground resolution as well as the accuracy of the results, obtained in both measurement sessions, for the whole surface of the rows of grapes. From the maps of NDVI indices the numerical values elaborates at the dates of July and September 2017 have been respectively equal to 0.831 and 0.949 .

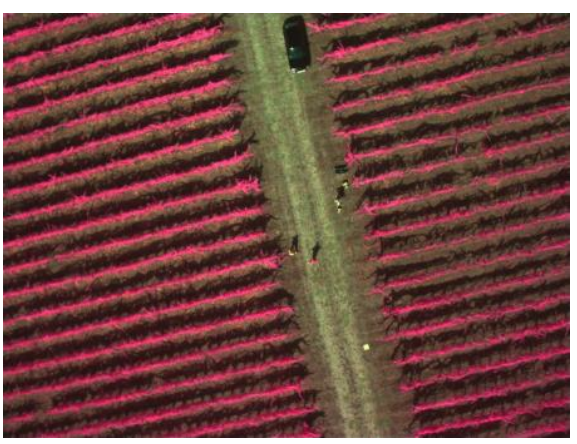

Figure 18. Ortophoto from multi-spectral image July $31^{\text {st }} 2017$

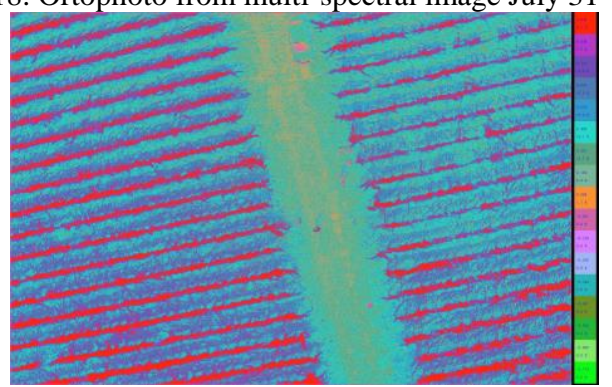

Figure 19. Ortophoto with NDVI values July $31^{\text {st }} 2017$

\subsection{Analysis of the results}

From the analysis of the results it can be concluded that the increase of the NDVI index of about $12 \%$ estimated during the monitoring period from June 2015 to September 2016 is indicative of the response of the vineyards to different climatic conditions and in accordance with the corrective remedies provided to the producer following the monitoring activities. This response is also correlated with the type of soil, strong clay matrix, therefore characterized by a significant water retention.

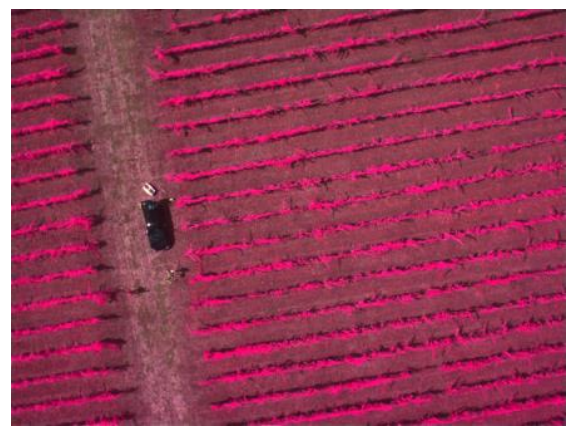

Figure 20.Ortophoto from multispectral image Sept. $2^{\text {nd }} 2017$

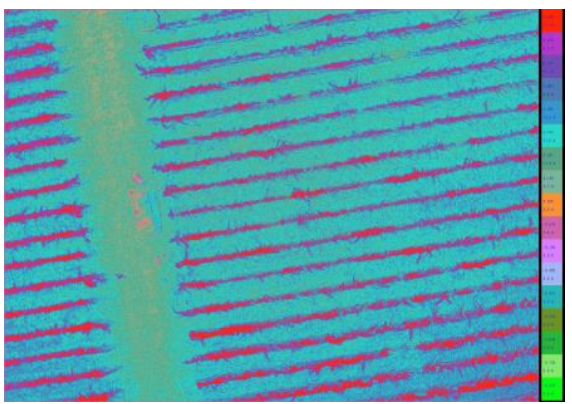

Figure 21. Ortophoto with NDVI values September $2^{\text {nd }} 2017$ 
In fact from a meteorological point of view, rainfall in the year 2015 , more copious than the year 2016, resulted in greater leaf production at a disadvantage of the quantity and quality of berries. Moreover the soil with a strong clay component, retaining rainwater by swelling, tends to decrease the amount of sugars present within the berries. The growth of the vineyard improves during the year 2016, characterized by a modest rainfall. In fact, a high rainfall is responsible for the formation of pests and mushrooms in the vines, and therefore no antiparasitic and anticritogamic treatment was practiced in 2016; moreover, the less water content retained by soil clay has allowed the higher sugar content of the acines, improving the alcoholic potential of the grape destined for vinification.

Conversely the mild decrease, equal to $4.6 \%$, of the NDVI index, recorded between the surveys of september 2016 and september 2017, is due to the high heat stress during the summer 2017 characterized by seasonal values of temperature higher than the average with peaks of $35^{\circ} \mathrm{C}$ and high humidity level. The table containing the definite values of NDVI index during the monitoring three years, as well as some conclusive considerations and recommendations for an optimal use of this innovative technique, are reported in the sequel.

\begin{tabular}{|c|c|}
\hline Monitoring & Value NDVI index \\
\hline June 2015 & 0,541 \\
\hline July 2015 & 0,637 \\
\hline July 2016 & 0,715 \\
\hline September 2016 & 0,995 \\
\hline July 2017 & 0,831 \\
\hline September 2017 & 0,949 \\
\hline
\end{tabular}

Table 3. NDVI indexes of monitoring 2015-2017

\section{CONCLUSIONS AND REMARKS}

Nowadays the real need to develop the growing precision farming sector is chiefly dictated by the need to improve production, achieving savings in terms of fertilizer and pesticide use, with a consequent reduction in environmental pollution and increased security for communities.

Through the use of combined information (data processed by photogrammetry by UAV and ground spectrometric measurements) the producer can uniquely determine which sector of his field presents real-time resolutions, such as the administration of major or minor quantities of fertilizers and / or higher irrigation cycles.

One of the most critical issues of this brand-new surveying methodology was mainly found in the flight autonomy of the UAV aircraft and the ability to install on board low-cost sensors rather than high-performance sensors. To avoid the risk of blurry and unclear images, it is advisable to use cameras/sensors equipped with electronic mechanisms known as a "rolling shutter" that allows the aircraft to move at lower speeds with respect to another mechanism known as a "global shutter". However, the "rolling shutter" allows one to obtain higher values ground resolution and even more accurate images without the risk of blurring.

A further constraint found during the measurement campaigns was the limited number of spectral bands provided by the multispectral cameras which did not allow estimation of certain vegetation parameters, such as moisture content.

For this reason, it would be desirable in the future to acquire data with hyper-spectral sensors.
Given the first results obtained with this study, more extensive and intensive field monitoring sessions in the future will be able to be used to determine how many samples of vines measured by the proposed methodology can become representative of the health status of vineyards and how they can be linked to the conditions of the ground.

The know-how gained with these first results permits to suggest that in the future the emergence of UAVs from geomatics technical digital photogrammetry can provide prescription maps for viticulture and provide recommendations to improve the efficiency of management in terms of quality, productivity and sustainability.

\section{BIBLIOGRAPHY}

Aasen A., Burkart A., Bolten A., Bareth G., (2015), Generating 3D hyperspectral information with lightweight UAV snapshot cameras for vegetation monitoring: From camera calibration to quality assurance. ISPRS Journal of Photogrammetry and Remote Sensing, 108, pp.245-259. doi: 10.1016/j.isprsjprs.2015.08.002

Bareth G., Bolten A., Gnyp M. L., Reusch S., Jasper J. (2016), Comparison of uncalibrated RGBVI with spectrometer - based NDVI derived from UAV sensing system on field scale. The International Archives of the Photogrammetry, Remote Sensing and Spatial Information Sciences, doi:10.5194/isprsarchivesXLI-B8-837-843; XXIII ISPRS Congress,12-19 July 2016, Praga , Repubblica Ceca

Bending J., Bolten A., Bareth G. (2012), Introducing a low cost mini - UAV for thermal - and multispectral - imaging. The International Archives of the Photogrammetry, Remote Sensing and Spatial Information Sciences Vol- XXXIX-B1-345; XXII ISPRS Congress, 25 Aug-01- Sept.2012, Melbourne, Australia

D’Urso M.G., Marino C.L. (2016), An application of close - up photogrammetry in viticulture. . The International Archives of the Photogrammetry, Remote Sensing and Spatial Information Sciences, doi:10.5194/isprsarchives XLI - B8,1243-1250- 2016 XXIII ISPRS Congress, 12-19 July 2016, Praga Repub. Ceca

Hadas E.,Jozkow G., Walicka A.,A.Borkowski,(2018), Determining geometric parameters of agricultural trees from laser scanning data obtained with unmanned aerial vehicle. The International Archives of the Photogrammetry, Remote Sensing and Spatial Information Sciences, Vol. XLII-2, ISPRS TC III

Krohnert M., Anderson R., Bumberger J., Dietrich P., Harpole W.S., Maas H.G., (2018), Watching grass grow - a pilot study on the suitability of photogrammetric techniques for quantifying change in aboveground biomass in grasland experiments. The International Archives of the Photogrammetry, Remote Sensing and Spatial Information Sciences, Vol. XLII-2, ISPRS TC III

Skarlatos D.,Vlachos M., (2018), Vegetation removal from UAV derived DSM, using combination of RGB and NIR imagery.ISPRS Annals of the Photogrammetry, Remote Sensing and Spatial Information Sciences, Vol. IV-2, ISPRS TC III

Tavakoli H., Mohtasebi S.S., Alimardani R., Gebbers R. (2014), Evaluation of different sensing approaches concerning to nondestructive estimation of leaf area index (LAI) for winter wheat. International Journal on Smart Sensing and Intelligent Systems vol. 7, n. 1, March 2014 
Tsouvaltsidis C., Zaid Al Salem N., Benari G., Vrekalic D., Quine B. (2015), Remote spectral imaging using a low cost UAV system. The International Archives of the Photogrammetry, Remote Sensing and Spatial Information Sciences; doi:10.5194/isprsarchives, XL-1-W4-25-31, International Conference on Unmanned aerial Vehicles in Geomatics, 30 August-02 September 2105, Toronto, Canada

Yang H., Li X., (2018), A band selection method for high precision registration of hyperspectral image. The International Archives of the Photogrammetry, Remote Sensing and Spatial Information Sciences, Vol. XLII-3, ISPRS Mid Term. TC III 